\title{
Model Development and Energy Management Control for Hybrid Electric Race Vehicles
}

\author{
K. Reeves \\ Motorsport Engineering, National Motorsport Academy \\ Nottingham, UK, kieran@ motorsport.nda.ac.uk
}

\author{
A. Montazeri and C.J. Taylor \\ Engineering Department, Lancaster University, UK \\ a.montazeri@lancaster.ac.uk, c.taylor@lancaster.ac.uk
}

\begin{abstract}
A Hybrid Electric Vehicle longitudinal dynamics model for the control of energy management is developed. The model is implemented using Simulink ${ }^{\circledR}$ and consists of a transitional vehicle speed input parameterized by, for example, the New European Driving Cycle. It is a backward looking model in that engine and motor on/off states are determined by the controller, dependent on wheel torque requirements and output targets. The objective of the simulation is to calculate tractive effort and resistance forces to determine longitudinal net vehicle force at the road. This article addresses model development and initial investigations of its dynamic behaviour in order to establish appropriate energy management strategies for the Hybrid Electric system. In particular, All Wheel Drive, Front Wheel Drive and Rear Wheel Drive drivetrain architectures are evaluated to determine minimum fuel usage and battery state of charge. The use of a logic controller allows a reduction of simulation time and ensures accurate results for charge depletion and harvesting. Simulated fuel consumption is within $1 \%$ of actual usage.
\end{abstract}

Keywords- Hybrid Electric Vehicles; Longitudinal Dynamics; Simscape; Vehicle Dynamics; Energy Management; Control; Race Vehicle.

\section{INTRODUCTION}

Simulation models are the focus for the majority of the automotive industry especially within the R\&D sector. Current vehicle dynamics simulation platforms do not allow for closedloop active control of vehicle stability. Simulation platforms such as Adams Car and GT-Drive require third party coupling to software such as Simulink ${ }^{\circledR}$ to enable control of the vehicle and, usually, pre-defined vehicle speed and cornering data are used as maxima for peak forces. In this regard, advanced controlled co-simulations include use of e.g. fuzzy control [1]. Other authors have developed longitudinal only dynamic simulations for the purpose of optimizing energy management strategies for emission drive cycle testing e.g. [2-4].

Vehicle models are normally classed as one of two types: forward looking and backward facing [5-6]. The model in this article is backward looking in that engine and motor on/off states are determined by the controller dependent on wheel torque requirements and the output targets e.g. fuel consumption and battery state of charge (SOC). The objective of the simulation is to calculate tractive effort and resistance forces to determine longitudinal net vehicle force at the road. It is envisaged that a fully optimised closed-loop system will also include the lateral model of reference [5] and a combined energy management/stability control system. In the first instance, however, the present article utilises Simscape modelling elements in Simulink ${ }^{\circledR}$ to focus on the longitudinal dynamics, powertrain model and control performance.

Simulink® software has been used for its relative ease of exchanging drivetrain layouts, with the purpose of incorporating Hybrid-Electric powertrain and energy management control strategies, in order to investigate the behaviour of All-Wheel (AWD), Front Wheel (FWD) and Rear Wheel (RWD) Drive vehicles. Hence, section II of the article reviews longitudinal acceleration dynamics, resistive forces, tractive forces and net work at the tyre-road surface. Section III describes the powertrain model and preliminary baseline control strategy. Section IV describes the simulation results, with the conclusion presented in section $\mathrm{V}$.

\section{LONGITUDINAL DYNAMICS}

Longitudinal vehicle speed $V_{x}$ is determined from,

$$
\dot{V}_{x}=\frac{F_{t}-F_{r}}{M}
$$

where $M$ is the vehicle mass, $F_{t}$ is the tractive effort (total) and $F_{r}=R_{r}+F_{g}+D_{A}$ the total resistance, in which $R_{r}, F_{g}$ and $D_{A}$ are the rolling resistance of the tyre at the road surface, road grade resistance and aerodynamic drag respectively.

\section{A. Resistance Forces}

When a vehicle attempts to ascend an incline the mass of the vehicle creates a resistive force against the vehicle. The uphill grading resistance with a road angle $(\beta)$ is,

$$
F_{g}=M g \sin \beta
$$

where, in the present simulations, gravity $g=9.81 \mathrm{~m} / \mathrm{s}^{2}$. The rolling resistance of a vehicle is typically due to the tyre contact patch with the road and the hysteresis of the tyre compound and materials [7]. When a vehicle travels on hard road surface the tyre pressure distribution tends to deflect and the resultant reaction force relocates a distance $d$ from the centre of the wheel. The ground reaction to the intended forward motion is known as rolling resistant moment,

$$
T_{r}=P d
$$

where $P$ is the force acting at the centre of the wheel. To maintain wheel rotation, a force acting on the centre of the wheel is required and must balance the rolling resistant moment. The rolling resistant moment can be substituted for a 
horizontal force that is acting on the centre of the wheel but in the opposite direction than the wheel is moving:

$$
R_{r}=P r_{c}
$$

where $r_{c}$ is the rolling resistance coefficient and is primarily a function of the tyre properties and environmental conditions. These include tyre materials, structure, tread pattern, tyre pressure, temperature, road material and the road adhesion qualities (e.g. the presence of rain or spilled liquids). A typical value for a concrete or asphalt road is $r_{c}=0.013$ compared to 0.02 for a rolled gravel road say. For typical vehicle dynamics calculations it is sufficient to assume resistance is a linear function of speed. The Bosch Handbook [8] states that for a common vehicle with tyre pressures in a normal range, on concrete, travelling up to speeds of $128 \mathrm{~km} / \mathrm{h}$, the rolling resistance coefficient can be represented as follows:

$$
r_{c}=0.01\left(1+\frac{V_{x}}{160}\right)
$$

Hence, the total road resistance can be expressed as:

$$
R_{r}+F_{g}=M g\left(r_{c} \cos \beta+\sin \beta\right)
$$

Finally, the aerodynamic drag is a function of air density $\rho$, the vehicle body shapes co-efficient of drag $C_{D}$, the frontal area $A_{f}$ and the vehicle speed, as follows,

$$
D_{A}=0.5 \rho A_{f} C_{D}\left(V_{x}-V_{w}\right)^{2}
$$

The headwind speed $V_{w}$ can also influence the aerodynamic drag and is accounted for in equation (7) by utilising a positive velocity against the motion of the vehicle.

\section{B. Equations of Motion}

When a vehicle is travelling in a forward direction, the major forces applied externally to the vehicle are the rolling resistance of both the front and rear tyres, expressed as rolling resistance moment (3), up-hill climbing resistance (2), tractive effort of both front $F_{t f}$ and rear wheels $F_{t r}$ (zero for a nondriven axle) and the aerodynamic drag (7). Hence, the vehicle motion in this longitudinal direction derived from (1) is,

$$
M \dot{V}_{x}=\left(F_{t f}+F_{t r}\right)-\left(R_{r f}+R_{r r}+D_{A}+F_{g}\right)
$$

where $R_{r f}$ and $R_{r r}$ are the rolling resistance of the front and rear tyres respectively. Equation (8) is the sum of the resistive forces subtracted from the vehicles total tractive effort. To determine tractive effort, the normal load on the vehicles axles needs to be determined. The sum of all moments of forces about the centre point of the tyre and ground is used to determine the normal load on the front (9) and rear (10) axles:

$$
\begin{aligned}
& W_{f}=\frac{L_{b}}{L} M G \cos \beta-\frac{h_{g}}{L}\left(D_{A}+F_{g}+M g r_{c} \frac{r_{d}}{h_{g}} \cos \beta+\dot{V}_{x}\right) \\
& W_{r}=\frac{L_{a}}{L} M G \cos \beta-\frac{h_{g}}{L}\left(D_{A}+F_{g}+M g r_{c} \frac{r_{d}}{h_{g}} \cos \beta+\dot{V}_{x}\right)
\end{aligned}
$$

where $L$ is the wheelbase (m), $L_{a}$ and $L_{b}$ are the distances from centre of gravity to the front and rear axle centre lines respectively $(\mathrm{m})$, and $r_{d}$ is the effective radius of the tyre. The tyre to ground contact patch can only support up to a maximum: even a small amount over and tractive effort will cause the tyre to lose traction and the tyre will spin. This maximum is the frictional coefficient and is a product of the coefficient of adhesion on the road $\mu_{i}$ and the normal load.

Using equations (4) and (8), the maximum tractive effort for the front and rear tyres is expressed as follows,

$$
\begin{gathered}
F_{m f}=\frac{\mu_{i} M g \cos \beta\left[L_{b}+f_{r}\left(h_{g}-r_{d}\right)\right] / L}{1+\mu_{i} h_{g} / L} \\
F_{m r}=\frac{\mu_{i} M g \cos \beta\left[L_{a}+f_{r}\left(h_{g}-r_{d}\right)\right] / L}{1+\mu_{i} h_{g} / L}
\end{gathered}
$$

where $h_{g}$ is the height of the centre of gravity, and $\mu_{i}=\mu_{p}$ for acceleration and $\mu_{i}=\mu_{s}$ for braking (see Table 1). At any given moment, the maximum torque supplied from the internal combustion engine or an electric motor through the relevant transmission and drive-train components to the wheel should not exceed the tyre to ground cohesion, or wheel spin will occur. Average tractive effort for some illustrative road surface materials is shown in Table 1. Equations (11) and (12) are both utilised in the case of an AWD vehicle, whilst either are dismissed or set to zero in the FWD or RWD configurations.

TABle 1 Illustrative Tractive EfFort CoEfFicients

\begin{tabular}{|l|c|c|}
\hline \multicolumn{1}{|c|}{ Road Surface } & Peaking $\boldsymbol{\mu}_{\boldsymbol{\rho}}$ & Slipping $\boldsymbol{\mu}_{\mathbf{s}}$ \\
\hline Asphalt and concrete (dry) & $0.8-0.9$ & 0.75 \\
\hline Concrete (wet) & 0.8 & 0.7 \\
\hline Asphalt (wet) & $0.5-0.7$ & $0.45-0.6$ \\
\hline Gravel & 0.6 & 0.55 \\
\hline
\end{tabular}

TABle 2 Simscape Vehicle Body PARAMETERS

\begin{tabular}{|l|c|}
\hline Parameter (COG: Center of Gravity) & $\begin{array}{c}\text { Value or } \\
\text { Symbol }\end{array}$ \\
\hline Mass (kg) & $M$ \\
\hline Number of wheels on each axle (numerical) & 2 \\
\hline Position of COG relative to front axles (m) & $L_{a}$ \\
\hline Position of COG relative to rear axles (m) & $L_{b}$ \\
\hline Position of COG relative to ground (m) & $h_{a}$ \\
\hline Effective frontal cross-sectional area (m $\left.{ }^{2}\right)$ & $A_{f}$ \\
\hline Aerodynamic drag coefficient (dimensionless) & $C_{D}$ \\
\hline
\end{tabular}

\section{Simscape Implementation}

Vehicle dynamics longitudinal behaviour is modelled using four Simscape elements in Simulink ${ }^{\circledR}$, namely the vehicle body, gear box, differentials and tyres. These are connected via various additional mathematical blocks to simulate mechanical efficiencies and inertias. The vehicle model consists of the parameters as shown in Table 2, which are subject to change per drivetrain layout. The model also consists of inputs for horizontal motion, determined by the longitudinal force from the tyre, headwind $V_{w}$ and road incline $\beta$. The outputs are $V_{x}$, and the front and rear vertical loads, NF and NR respectively, where the latter are determined as follows,

$$
\begin{gathered}
N F=\frac{-h_{g}\left(D_{A}+M g \sin \beta+M \dot{V}_{x}\right)+L_{b} M G \cos \beta}{n\left(L_{a}+L_{b}\right)} \\
N R=\frac{h_{g}\left(D_{A}+M g \sin \beta+M \dot{V}_{x}\right)+L_{a} M G \cos \beta}{n\left(L_{a}+L_{b}\right)}
\end{gathered}
$$


where $n$ is the number of wheels per axle. The vehicle motion is determined by the net effect of all the forces and torques acting on it (8). The longitudinal tyre forces push the vehicle forward or backward. The weight $(M g)$ of the vehicle acts through its center of gravity. The longitudinal forces from the tyre that are applied to the body for forward motion are further developed through the Pacejka Magic Formula Tyre model [7]:

$$
F_{x}=F_{z} D \sin (C \arctan (B k-E(B k-\arctan (B k))))
$$

where $F_{z}$ is the vertical load on the tyre $(\mathrm{NF} / 2$ or NR/2) and $k$ is the wheel slip. The Magic Formula is characterised by four dimensionless coefficients $(B, C, D, E)$, i.e. stiffness, shape, peak and curvature. These depend on the curvature fit from the tyre manufacturer's data or the surface conditions, as shown in Table 3 . Wheel slip is determined by the difference in wheel hub longitudinal velocity $V_{h x}$ as opposed to slip velocity $V_{s x}$,

$$
V_{s x}=r_{w} \Omega-V_{h x}
$$

Slip velocities are a function of hub rotational velocity and tread velocity $r_{w} \Omega$,

$$
k=V_{s x}\left|V_{h x}\right|
$$

The tyre inputs are vertical force and axle rotation (from the powertrain via the drivetrain) and the outputs are longitudinal driving force for the vehicle body and tyre slip.

TABle 3 Pacejka Longitudinal Tyre CoEFFicients [7]

\begin{tabular}{|l|c|c|c|c|}
\hline Surface & $A$ & $B$ & $C$ & $D$ \\
\hline Asphalt (dry) & 10 & 1.9 & 1 & 0.97 \\
\hline Asphalt (wet) & 12 & 2.3 & 0.82 & 1 \\
\hline Snow & 5 & 2 & 0.3 & 1 \\
\hline Ice & 4 & 2 & 0.1 & 1 \\
\hline
\end{tabular}

The drivetrain is determined as the gearbox and differential(s) dependent on two or four wheel drive configurations. The gearbox provides the angular rotation and torque to the differential(s) from the input source (powertrain) of motor/generator and/or internal combustion engine. The gearbox allows an increase and reduction of rotational speed from the powertrain due to gear ratio allocation. The gearbox has an input $\left(\omega_{1}\right)$ and output $\left(\omega_{2}\right)$ of angular speed,

$$
\omega_{1}=N \omega_{2}
$$

where $N$ is the gear ratio. Further gear ratios can be applied as a final drive through the differential in a similar manner. A motor/generator unit and an internal combustion engine define the powertrain model. Look up tables determine power and torque profiles and fuel usage if so desired as a control target. The control system is designed to utilise power deployment to target stability in a future 6 degree-of-freedom model and lap performance of race vehicles. The present model concentrates on energy management and power deployment with regards to state of charge of the batteries and torque demand. Firstly the required forces and torque at the tyre(s) must be determined in order to ensure correct power deployment from the powertrain. This is determined from equations (7) and (8) as follows:

$$
F_{\text {reqtyre }}=M \frac{d V_{x}}{d t}+g M \sin \beta+g \cos \beta M\left(f_{1}+\frac{V_{x}}{f_{2}}\right)+D_{A}
$$

where $f_{l}$ and $f_{2}$ are inflation tire pressure offset and coefficient.

\section{POWERTRAIN AND CONTROL IMPLEMENTATION}

The powertrain components of the model detailed in this article are created using the Parallel Series hybrid electric vehicle model available from Mathworks [9], and combined by the authors with the relevant AWD, FWD, and RWD drivetrain systems. Vehicle dynamic motion and tyre forces are the main purpose of the model, hence a relatively straightforward powertrain model is used here. To provide adequate torque for throttle position in the preliminary experiments reported below, the SimDriveline Spark Ignition (SI) combustion engine model element was utilised. This is defined by capacity, type of ignition and maximum power, with look up tables for brake specific fuel consumption and power. The associated electrical subsystem illustrated in Fig. 1 comprises of a motor and generator [10]. The battery architecture is that of a NickelMetal-Hydride energy storage device, represented by the following discharge and charge models:

$$
\begin{aligned}
& D_{M}\left(i^{*}>0\right)=E_{0}-K \frac{Q}{Q-i t} i^{*}-K \frac{Q}{Q-i t} i t+\mathcal{L}^{-1}\left(\frac{A}{\frac{1}{(B i(t)) s+1}} 0\right) \\
& C_{M}\left(i^{*}<0\right)=E_{0}-K \frac{Q}{|i t|+0.1 Q} i^{*} K \frac{Q}{Q-i t} i t+\mathcal{L}^{-1}\left(\frac{A}{\frac{1}{(B i(t))_{s+1}} s} \frac{1}{s}\right)
\end{aligned}
$$

in which $E_{0}$ is a constant voltage $(\mathrm{V}), K$ is the polarization constant $(\mathrm{Ah}-1)$ or Polarization resistance (Ohms), $i^{*}$ represents the low frequency current dynamics (A), it the extracted capacity (Ah), $Q$ the maximum battery capacity (Ah), $A$ exponential voltage $(\mathrm{V})$ and $B$ exponential capacity $(\mathrm{Ah})^{-1}$.

For effective control of power deployment from the electric machine or motor, the target result first has to be established. In the case of drive cycle testing for emissions, for example, it could be determined that fuel economy must be minimised whilst maintaining adequate state of charge to deploy the motor. Engine on/off parameters would need to be determined against minimum vehicle speeds and the motor-to-generator switching parameters for regenerative braking must be set for the braking events. In the case of a race vehicle maximum power deployment is the typical target whilst ensuring enough electrical energy can be harvested and stored during the simulation event. For either of these scenarios, control of the powertrain is necessary. In this regard, the four major events a vehicle encounters are: (i) start up and low speed; (ii) acceleration; (iii) braking and (iv) cruising. A fifth event for a race car could be included, namely high speed. However, if the gearing of a race vehicle is correct the race car should be accelerating and only reach velocity maxima as the braking zone begins. For this reason, high speed has been neglected.

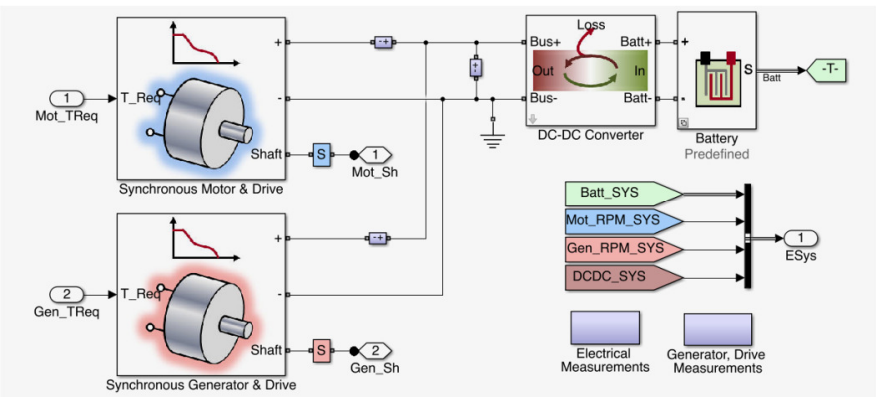

Fig. 1. Motor/Generator Model (Simscape Simulink® block diagram). 


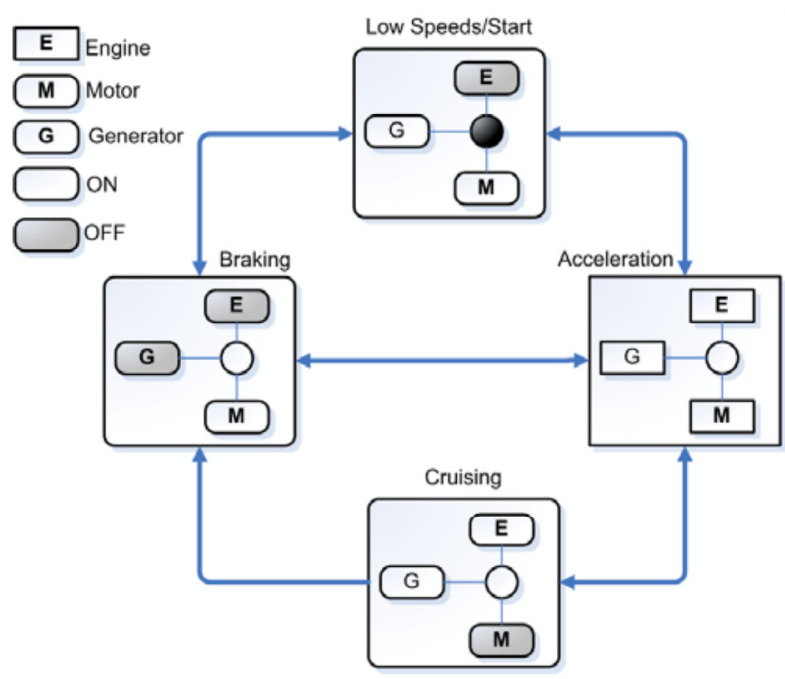

Fig. 2. Mode Logic for Road Vehicles [10].

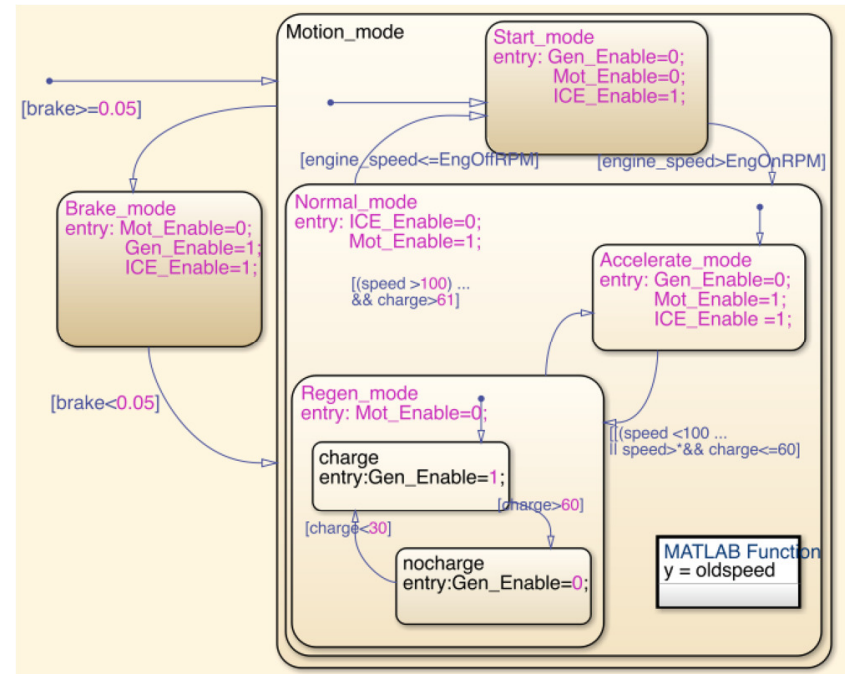

Fig. 3. Revised Stateflow ${ }^{\circledR}$ Mode Logic for Race Vehicle. Regeneration events for Braking and SOC $<60 \%$. Motor enabled above $100 \mathrm{~km}-\mathrm{h}$.

For each event above, power deployment scenarios to meet the torque demand are determined. Following the approach of Mahaptra et al. [10], Fig. 2 illustrates a power strategy for road vehicles. In the case of race vehicles, the diagram differs only in that the Motor is utilised at start up and low speed (pit lane) and the generator would be switched on during braking events to harvest the maximum energy during a lap, although braking stability and effectiveness must also be maintained.

Four controllers have been utilised to allow the vehicle to operate according to this mode logic: Engine speed; Motor Torque and Speed; Generator Torque and Speed; and Battery State of Charge (SOC). The simulation is set to target specific output parameters. For the initial simulation results in this article, the output criteria set is for the road vehicle to challenge the controller by minimising fuel usage whilst maintaining a SOC of $100 \%$. Here, charge produced from regenerative braking could be deployed. The mode logic was subsequently re-written for a race vehicle scenario. The desired speed data represents six laps of Brands Hatch, plus an out and in lap so that pit lane speed is included. Fig. 3 illustrates this latter modified Stateflow® Mode Logic, while Fig 8 (see later) shows the Brands Hatch speed data. The mode logic was set to utilise the motor only during low speed and deploy both the motor and combustion engine during on-track acceleration events. All braking events would utilise regenerative braking.

The race vehicle mode logic control was amended further to ensure the acceleration event was utilised much more often. In particular, the initial road vehicle scenario had an instruction set to be in cruise mode if the vehicle speed change was less than $+/-2 \%$ of the previous speed; however, this requirement was subsequently removed and cruise mode ignored. The regeneration event could only be utilised and was tested for 2 scenarios: energy sustaining and energy depleting. During an energy depletion scenario all electric power could be used until the battery store was zero, whereas in energy sustaining mode a minimum SOC percentage was specified as a rule. The power of the combustion engine would be increased from $114 \mathrm{~kW}$ at $5000 \mathrm{RPM}$ to $400 \mathrm{~kW}$ at $7300 \mathrm{RPM}$. The brake specific fuel consumption table was also amended to use more fuel. Finally, the weight of the race vehicle was reduced from $1200 \mathrm{~kg}$ of the road vehicle to $1130 \mathrm{~kg}$ and the tyre radius modified to $0.35 \mathrm{~m}$.

\section{RESULTS}

Simulation experiments for the road vehicle are completed over the three different drivetrain designs, i.e. AWD, FWD and RWD. The vehicle and powertrain parameters (Table 4) remained unchanged across all vehicles with the only variation relating to the additional resistive forces and frictions within the extra differential and driven tyres in the AWD case.

TABle 4 POWERTRAin \& Vehicle Parameters FOR THE DRIVETRAIN ARCHITECTURE (ALL VEHICLES)

\begin{tabular}{|c|c|}
\hline Battery & Value \\
\hline Maximum Capacity (Ah) & 8.1 \\
\hline Fully Charged Voltage (V) & 232.8 \\
\hline Nominal Discharge Current (A) & 3.52 \\
\hline Internal Resistance (Ohms) & 0.25 \\
\hline Capacity (Ah)@ Nominal Voltage & 7.33 \\
\hline \multicolumn{2}{|l|}{ Motor/Generator } \\
\hline Torque $(\mathrm{Nm})$ & 400 \\
\hline Efficiency $(\%)$ & 90 \\
\hline Efficiency measurement speed (RPM) & 2000 \\
\hline Efficiency measurement torque (Nm) & 200 \\
\hline \multicolumn{2}{|l|}{ Internal Combustion Engine } \\
\hline Type & SI \\
\hline Maximum Power $(\mathrm{kW})$ & 114 \\
\hline Speed @ Maximum Power (RPM) & 5000 \\
\hline Maximum Speed (RPM) & 6000 \\
\hline Stall Speed (RPM) & 500 \\
\hline \multicolumn{2}{|l|}{ Vehicle Parameters } \\
\hline Weight $(\mathrm{kg})$ & 1200 \\
\hline Mass Distribution (front:rear) & $50: 50$ \\
\hline Drag Coefficient & 0.26 \\
\hline Tyre Radius (m) & 0.3 \\
\hline Wheel Inertia $\left(\mathrm{kg}-\mathrm{m}^{2}\right)$ & 0.1 \\
\hline
\end{tabular}


This consistency allows appropriate analysis of the results regarding powertrain deployment due to additional drivetrain features only. Furthermore, control targets are compared without additional complexities having to be included to compensate for weight increases on various axles and vehicle overall weight. This would determine the suitability of the mode logic model and appropriate control behaviour.

The New European Driving Cycle (NEDC) determined the required vehicle speed. Fuel usage was set to a maximum of 0.25 litres for the entire 1180 second drive cycle. As would be expected, Fig. 4 shows that the additional forces required to propel an AWD drivetrain meant that this system used the most fuel, whilst the FWD system used the least amount of fuel across the entire drive cycle. The RWD system has the added complexity of a prop-shaft and power direction changes of 90 degrees from engine to prop-shaft and back again through 90 degrees via the differential to the wheels, hence the RWD system has fuel use closer to that of the AWD system. The controller ensured no drivetrain architecture used more than $0.25 \mathrm{~L}$, hence all systems differential fuel usage was negligible in absolute terms, with the AWD, RWD and FWD systems using $0.242 \mathrm{~L}, 0.241 \mathrm{~L}$ and $0.240 \mathrm{~L}$ over the NEDC respectively.

The State of Charge (SOC) of the battery for all three drivetrain architectures operated within a window of $99 \%$ charge to $103 \%$ as illustrated in Fig. 5. The regeneration during braking meant a charge could be stored (greater than 100\%) and deployed in the traction events to ensure required accelerations were met. As the battery could maintain a minimum of $99 \%$ charge, the mode logic for Normal mode was set so that the engine was not enabled and the cycle could be carried out on electric energy only. As a $400 \mathrm{Nm}$ motor was utilised the vehicle could complete the test in electric only mode and sustain a battery charge of $99-102 \%$ due to the amount of deceleration events in the NEDC. Hence, to conclude, the mode logic model behaves appropriately with regard to drive modes and maintaining target parameters.

The mode logic was subsequently replaced with that described above for the race vehicle and in a RWD configuration. The first simulation experiment was set for charge depleting. In this case, the vehicle data are taken directly from a Cosworth Pi Sigma data logger. The vehicle has an initial 83 seconds sat in the pit garage but as soon as the vehicle starts to move the motor is used for pit lane exit. In this instance, with a $400 \mathrm{Nm}$ motor, by the time the engine is enabled and the vehicle accelerates down the first straight, the battery power has depleted, as shown by Fig 6 . The vehicle subsequently attempts to recover for the remainder of the session but, as the motor was deployed for all acceleration events and the controller set for charge depletion, the regenerative braking system would not suffice to provide enough electrical storage for the entire session.

For the second simulation experiment, the mode logic was replaced with one that would only utilise the motor during acceleration events when the vehicle speed was above $100 \mathrm{~km} / \mathrm{h}$. This allowed the motor to assist with all acceleration events but was switched off for low speed and pit lane use, where regeneration is difficult. The desired vehicle speed data was amended to remove the pit lane speeds and out-lap events, so that the energy management could be assessed over four flying laps. With this new mode logic, illustrated in Fig 3, the generator was set to work during braking events whilst the motor would switch off during an acceleration event if the battery fell below $60 \%$ charge. As the data was taken from a race vehicle, the lap time delta from slowest to fastest lap was 0.8 seconds, and the battery usage per lap was between $4.2 \%$ and $4.7 \%$, with the main battery usage being used through the long medium speed final corner and start/finish straight, as illustrated in Fig 7. A 20-lap race could be completed with this control logic.

The final part of the test shown in Fig. 7 is to include an inlap where braking force is greatly reduced due to the slower speed of the vehicle. During this period the battery was used much more and the control switched to regenerative mode as the SOC fell below $60 \%$. In this case, the battery SOC managed to recover $8 \%$ during the final braking events when the motor was switched off. Finally, the Brands Hatch vehicle speed data set used for these experiments is shown in Fig. 8. Although not used in its entirety for the illustrative simulation experiments above, the data available to the authors for this and future research includes pit lane exit, out-lap, six flying laps, in-lap and pit lane entry. In Fig. 8, zero speed represents the stationary vehicle in the pit garage.

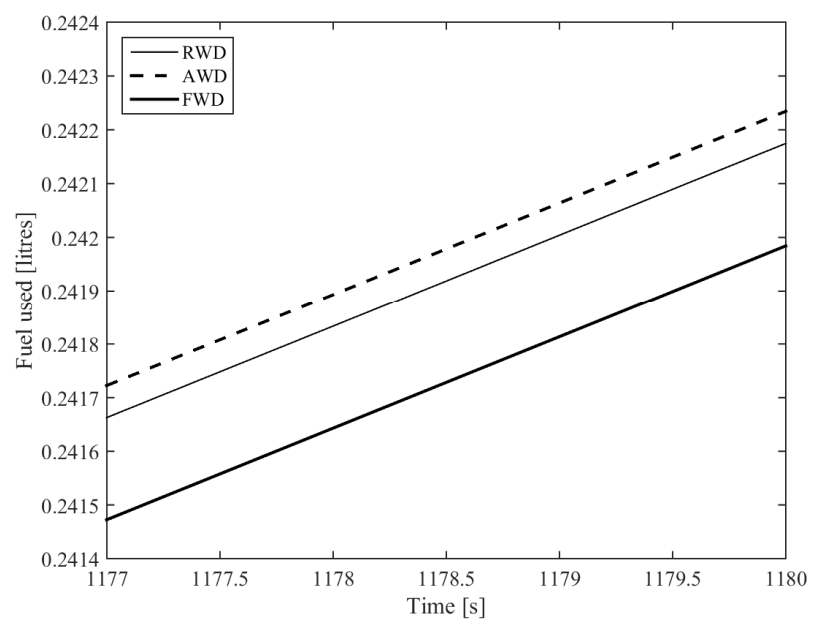

Fig. 4. Fuel used by AWD, RWD and FWD vehicles at end of cycle.

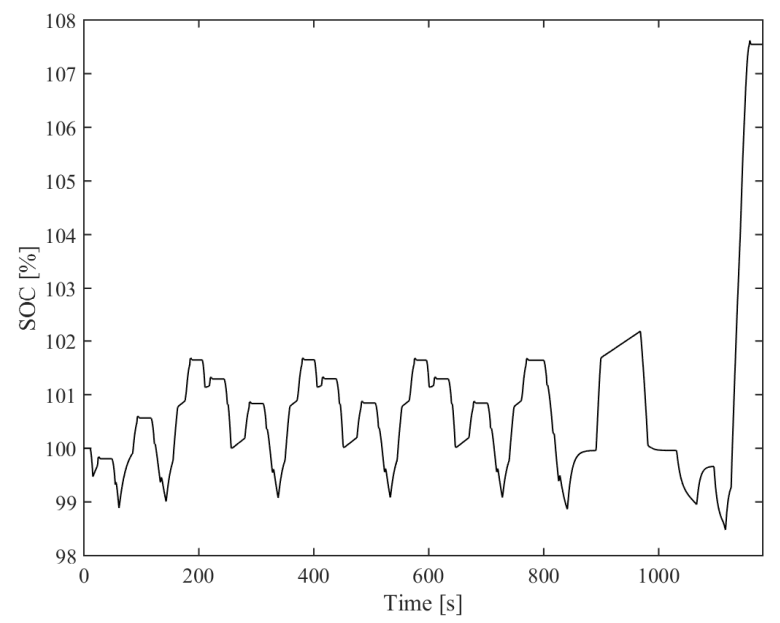

Fig. 5. Road vehicle SOC for using a charge sustaining mode target of $100 \%$. 


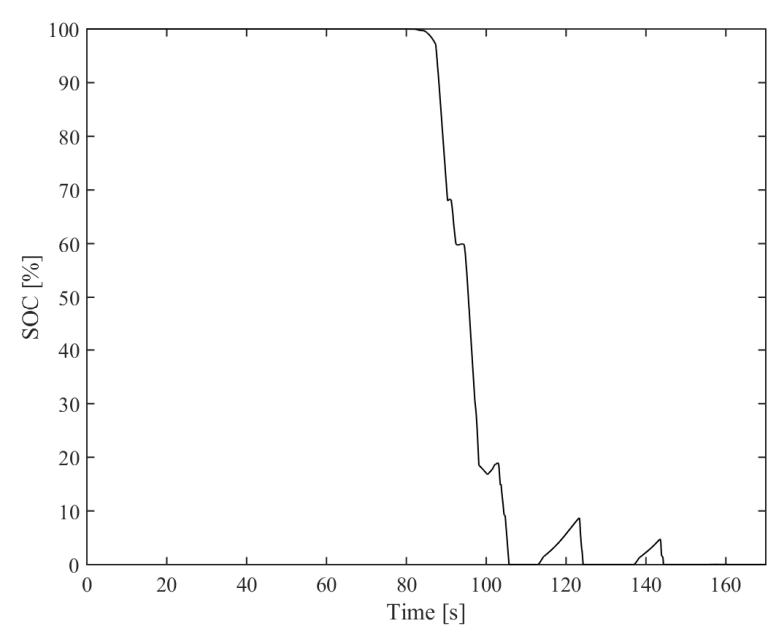

Fig. 6. Race Vehicle battery storage SOC using charge depletion mode.

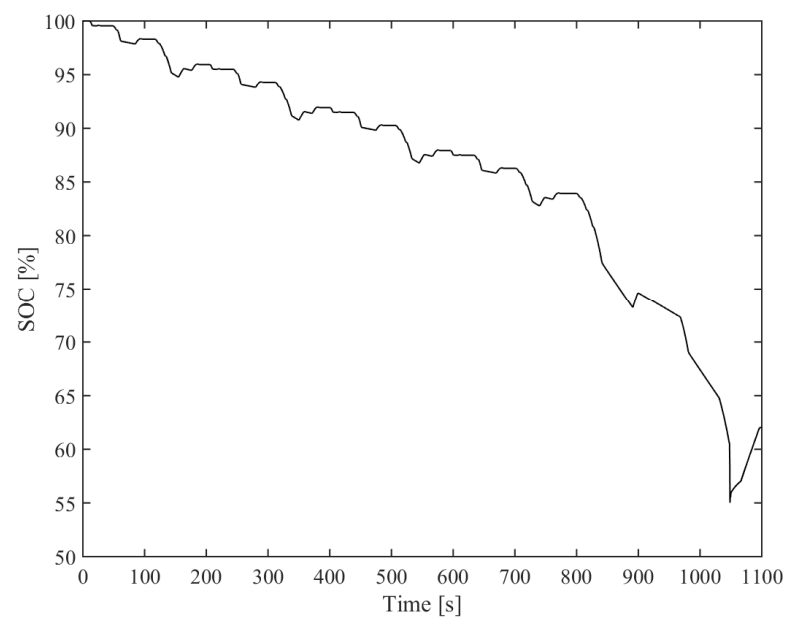

Fig. 7. Race Vehicle battery storage SOC with four flying laps and an in lap.

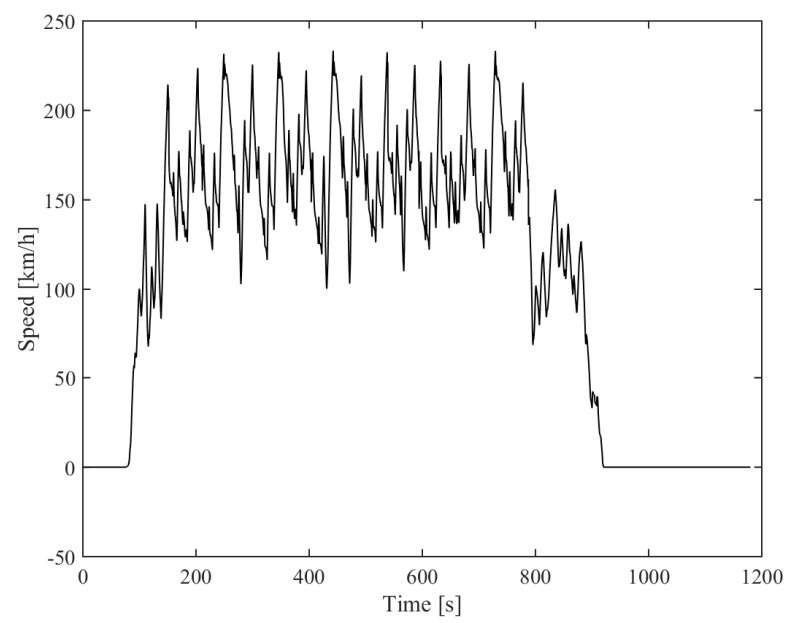

Fig. 8. Brands Hatch vehicle speed data.

\section{CONCLUSIONS}

This article has described the development and software implementation of a (backward looking) Hybrid Electric Vehicle dynamics model. It shows that the longitudinal dynamics can be realistically simulated using readily available Simscape model components in Simulink ${ }^{\circledR}$ but with the added value of incorporating appropriate Hybrid Vehicle behaviour. Suitable responses to control strategies for energy management can be generated without the need for detailed electrical models or the additional complexity of the power and drive train systems. This new simulation will allow subsequent integration with a lateral dynamic model [5] incorporating stability control that can target energy efficiencies and power deployment strategies, all with conveniently fast simulation timeframes. In this regard, the authors are presently investigating the use of State-Dependent [11] and Genetic Algorithm [12] optimization and control methods, and this will be reported in future articles.

The model provides an effective tool for maximizing race lap times (with deployment of the motor and stability handling targets) whilst simultaneously managing energy harvesting. The simulation tool can also be used for powertrain component size identification. In this regard, a plethora of motor and generator sizes can be tested to establish performance contributions and appropriate regeneration benefits. Finally, battery storage devices can also be analysed. In this manner, detailed energy management strategies can be established and tested through simulation for a range of practical scenarios prior to track testing, and this is the focus of on-going research by the authors.

\section{REFERENCES}

[1] S. Li and L. He, "Co-simulation of Vehicle ESP System Based on ADAMS and MATLAB" Journal of Software, Vol. 6, No. 5, pp. 866872, May 2011.

[2] D.W. Gao and A. Emadi, "Modelling and Simulation of Electric and Hybrid Vehicles" Proceedings of the IEEE, Vol. 95, No. 4, pp. 729-745, April 2007.

[3] F. Yan, J. Wang and K. Huang, "Hybrid Electric Vehicle Model Predictive Control Torque-Split Strategy Incorporating Engine Transient Characteristics" IEEE Transactions on Vehicular Technology, Vol. 61, No. 6, pp. 2458-2467, July 2012.

[4] T. Hofman, M. Steinbuch, R. van Druten and A. Serrarens, "Rule-based energy management strategies for hybrid vehicles" International Journal Electric and Hybrid Vehicles, Vol. 1, No. 1, pp. 71-94, 2007.

[5] K. Reeves, A. Montazeri and C. J. Taylor, "Validation of a Hybrid Electric Vehicle dynamics model for energy management and vehicle stability control". IEEE International Symposium on Industrial Electronics, Santa Clara, California, USA, 2016.

[6] K. B. Wipke, M. R. Cuddy and S. D. Burch "ADVISOR 2.1: A userfriendly advanced powertrain simulation using a combined backward/forward approach" IEEE Transactions on Vehicular Technology, Vol. 48, No. 6, pp. 1751-1761, Nov 1999.

[7] H. B. Pacejka, Tyre and Vehicle Dynamics. 2nd ed. Oxford: Butterworth-Heinemann. p61-64. 2005.

[8] R. Bosch, Bosch Automotive Handbook. 8th ed. Cambridge (MA): Bentley Publishers. 2014.

[9] S. Miller, Hybrid-Electric Vehicle Model in Simulink. 2010. Available: http://uk.mathworks.com/matlabcentral/fileexchange/28441-hybridelectric-vehicle-model-in-simulink. Last accessed 22nd Feb 2016.

[10] S. Mahapatra, T. Egel, R. Hassan, R. Shenoy and M. Capone, "ModelBased Design for Hybrid Electric Vehicle Systems" The Mathworks Inc. 2008 (2008-01-0085).

[11] C. J. Taylor, P. C. Young and A. Chotai, True Digital Control: Statistical Modelling and Non-Minimal State Space Design, John Wiley \& Sons Ltd., 2013.

[12] A. Montazeri and J. Poshtan, "GA-based optimization of a MIMO ANC system considering coupling of secondary sources in a telephone kiosk" Applied Acoustics, Vol. 70, No. 7, pp. 945-953, 2009. 